\title{
Cloud Deployment Methods In Guarantee of Protection and Confidentiality Constraints
}

\author{
Sarfaraz Ahmed $^{1}$, T. Senthil Kumaran ${ }^{2}$ \\ ${ }^{1}$ Research Scholar, Department of Information Technology, AMET University, Chennai \\ ${ }^{2}$ Associate Professor, Department of Computer Science, ACS College of Engineering, Bangalore
}

\begin{tabular}{l} 
Article Info \\
\hline Article history: \\
Received Jul 29, 2017 \\
Revised Nov 24, 2017 \\
Accepted Dec 19, 2017 \\
\hline
\end{tabular}

Keywords:

Cloud Deployment

Confidentiality

Guarantee

Protection

\begin{abstract}
Despite of the few advantages of moving venture basic resources for the Cloud, there are difficulties particularly identified with protection and confidentiality. It is essential that Cloud Users comprehend their protection and confidentiality needs, in view of their particular setting and select cloud demonstrate best fit to bolster these requirements. The writing gives works that emphasis on examining protection and confidentiality issues for cloud frameworks however such works don't give a methodological way to deal with evoke security and security necessities neither one of the methods to choose cloud arrangement models in light of fulfillment of these prerequisites by Cloud Service Providers. This work progresses the present cutting edge towards this bearing. Specifically, we consider necessities designing ideas to inspire and investigate protection and confidentiality prerequisites and their related instruments utilizing a calculated structure and an efficient procedure. The work presents confirmation as proof for fulfilling the protection and confidentiality necessities regarding culmination and reportable of security occurrence through review. This enables point of view cloud clients to characterize their affirmation prerequisites so that proper cloud models can be chosen for a given setting.
\end{abstract}

Copyright $@ 2018$ Institute of Advanced Engineering and Science. All rights reserved.

\section{Corresponding Author:}

Sarfaraz Ahmed,

Research Scholar, Department of Information Technology,

AMET University, Chennai

\section{INTRODUCTION}

Protection and confidentiality are significant worries for associations, which block cloud adaption as relocating into the cloud implies associations need to store their touchy electronic resources into the suppliers' foundation [1]. Existing business applications and information are for the most part controlled through the supplier's foundation relying upon the picked demonstrate, i.e. Saas, PaaS, IaaS, on which clients might not have full/any control. Clients' information is for the most part put away in a multi-inhabitant stage. This situation presents additional protection and confidentiality challenges contrasting with the customary registering condition [2].

It contributes to the current state of the art by providing a modeling framework that supports the elicitation and analysis of security and privacy needs, and a cloud migration process for the selection of an appropriate cloud model [3]. Secondly, it introduces assurance requirements in the proposed framework and in the designed process and it examines their critical role during the migration process for the selection of the most appropriate Cloud Service Provider (CSP) [4]. Specifically, we use requirements engineering concepts such as goal, actor, security and privacy constrains, mechanisms and we introduce assurance requirement to obtain evidence for the satisfaction of the requirements through audit and transparency. This allows us on one hand to identify and analyze security and privacy requirements and on the other hand to verify whether a chosen cloud deployment model addresses the identified requirements with appropriate mechanisms based on a specific organizational context [5]. 
It adds to the present cutting edge by giving a demonstrating system that backing the elicitation and examination of protection and confidentiality needs, and a cloud movement prepare for the determination of a fitting cloud display [6]. Besides, it presents confirmation prerequisites in the proposed structure and in the composed procedure and it inspects their basic part amid the movement procedure for the determination of the most fitting Cloud Service Provider (CSP). In particular, we utilize necessities building ideas, for example, objective, performing artist, protection and confidentiality obliges, systems and we acquaint confirmation prerequisite with get prove for the fulfillment of the necessities through review and straightforwardness [7]. This enables us on one hand to recognize and investigate protection and confidentiality necessities and then again to check whether a picked cloud sending model addresses the distinguished prerequisites with fitting instruments in view of a particular hierarchical setting [8]. The black hole attacks occurred in routing and the effects of performance of geographical routing in MANET is described in [9]. Based on new cryptographic scheme a secured and protected information scenario is generated using digital signature [10]. This technique is based on user authentication and security mechanism. The data holder needs to send the new figure content to the cloud, while the cloud just replaces the obsolete figure message and does not have to exchange it to the non-denied clients, so the extra correspondence costs is information [11].

\section{PROPOSED SYSTEM}

The novelty of the proposed displaying dialect is the way that it joins ideas from the necessities engineering, cloud computing, protection, confidentiality and auditing area. It utilizes new ideas, for example, cloud client, cloud service provider, audit, and instrument, which are important to evoke and examination of necessities and checks confirmations to bolster these prerequisites in light of authoritative setting. The meta-model of the dialect characterizes all ideas.

The focal idea of the proposed dialect is that of a performer, which speaks to an element that has key objectives and goals inside a framework or a hierarchical setting. A performing artist can be human, a framework, or an association. For our situation, association, cloud client and cloud service provider are three unique sorts of performing artists. A cloud client on-screen character can be individual or association who needs cloud administration and organization model to bolster its particular key objective and expectation. A cloud service provider performing artist has two novel properties, i.e., administration and deployment model to bolster the cloud clients. The performer association setting considers the extent of the authoritative elements, for example, objective, administrations, and foundation and incorporates movement needs into cloud that ought to be upheld by a cloud specialist organization.

The identification and analysis of the respective organization's security and privacy requirements is conducted. Security manager and internal audit (if any) are mainly involved for this activity. Two steps and two respective outcomes are defined, the Security and Privacy requirement identification and deployment scenario description.

Once the pertinent protection and confidentiality objectives and cloud relocation needs have been distinguished, an elicitation and examination prepare for protection and a confidentiality prerequisite is utilized. We construct our examination in light of the ideas of protection and confidentiality prerequisites, characterized in the introduced meta-model, to empower designers to enough catch protection and confidentiality necessities. Protection and confidentiality prerequisites are evoked considering association substances, for example, association objective, performing artists, cloud movement needs, dangers and vulnerabilities. In addition, authoritative particular record, for example, hierarchical approaches, objectives, and business forms, outer sources, (for example, laws and controls, conceivable outside dangers distinguished), and applicable innovative limitations in light of the innovation utilized, (for example, imperatives that may be special for distributed computing situations) can likewise be utilized to evoke the necessities.

The distinguished necessities are dissected in light of the potential dangers and vulnerabilities of the CSP surface and its encompassing condition. Subsequently, this progression likewise incorporates recognizable proof of dangers and vulnerabilities to investigate the necessities for further refinement. It is likewise significant that protection and confidentiality prerequisites are the same independent of particular cloud organization models. A sending situation is recognized and portrayed. The portrayal depends on data identified with the organization model to be utilized, the facilitating model, the pertinent administrations and assets to be sent alongside the accessible protection and confidentiality components. 


\section{DISCUSSION}

Trust basically works in a best down manner, as each layer needs to believe the layer promptly underneath it, and requires a security ensure at an operational, specialized, procedural and lawful level to empower secure correspondences with it. A trusted testament fills in as a solid electronic "travel permit" that sets up a substance's character, qualifications and obligations. Trust can be seen as a chain from the end client, to the application proprietor, who thus confides in the framework supplier (either at a virtual or equipment level as per the chose benefit display). A Trusted Third Party can give the required trust by ensuring that imparting parties are who they claim to be and have been examined to hold fast to strict necessities. This procedure is performed through the affirmation procedure, amid which an element requiring confirmation is required to comply with an arrangement of strategies and necessities. TTP is a perfect security facilitator in an appropriated cloud condition where elements having a place with partitioned authoritative spaces, with no earlier information of each other, require building up secure communications.

\section{CONCLUSION}

Cloud relocation is a standout amongst the most critical concerns these days for both private and open associations since because of the current budgetary circumstances each association is pointing on cost decreases without losing proficiency and administration quality. Notwithstanding, before relocating administrations, information or foundation into the cloud, it is important to acknowledge and comprehend the movement needs and dangers that cloud relocation ruins. These dangers change among associations particularly because of the changeability of data and in addition the kind of cloud administrations every association wishes to utilize. At last, the determination of the separate cloud display that will be received assumes a critical part on the potential dangers that the association may confront too. In this way, the part of security and protection are essential for an association to choose which cloud arrangement fits best its needs and prerequisites.

\section{REFERENCES}

[1] Treacy B C, Bruening P J. Privacy \& Security Law Report: Privacy. Security Issues Raised by Cloud Computing. The Bureau of National Affairs. 2009.

[2] Dorey P G, Leite A. Commentary: Cloud computing-A security problem or solution? Information security technical report. 2011; 16. (3): 89-96.

[3] Grobauer B, Walloschek T, Stocker, E. Understanding cloud computing vulnerabilities. IEEE Security \& Privacy. 2011; 9(2): 50-57.

[4] Davison R, Martinsons M G, Kock N. Principles of canonical action research. Information systems journal. 2004; 14(1): 65-86.

[5] Takabi H, Joshi J B, Ahn G J. (). Security and privacy challenges in cloud computing environments. IEEE Security \& Privacy. 2010; 8(6): 24-31.

[6] Mulazzani M, Schrittwieser S, Leithner M, and Huber M, Weippl E R. Dark Clouds on the Horizon: Using Cloud Storage as Attack Vector and Online Slack Space. In USENIX security symposium. 2011: 65-76.

[7] Jamshidi P, Ahmad A, Pahl C. Cloud migration research: a systematic review. IEEE Transactions on Cloud Computing. 2013; 1(2): 142-157.

[8] Mouratidis H, Kalloniatis C, Islam S, Huget M P, Gritzalis S. Aligning Security and Privacy to Support the Development of Secure Information Systems. J. UCS. 2012; 18(12): 1608-1627.

[9] Shanthi H J, Anita E M. Performance analysis of black hole attacks in geographical routing MANET. 2014.

[10] Ganeshkumar K, Arivazhagan D., Generating a digital signature based on new cryptographic scheme for user authentication and security. Indian Journal of Science and Technology. 2014; 7(S6): 1-5.

[11] Krishna Keerthi Chennam, and M. Akka Lakshmi., Cloud Security in Crypt Database Server Using Fine Grained Access Control. International Journal of Electrical and Computer Engineering (IJECE). 2016; 6(3); 915 924. 